\title{
Tradução e Adaptação Cultural do Modified-University of California at Los Angeles Shoulder Rating Scale para a Língua Portuguesa
}

\section{Translation and Cultural Adaptation of the Modified-University of California at Los Angeles Shoulder Rating Scale to Portuguese Language}

\author{
Elaine Cristina Oku ${ }^{(1)}$, Ana Paula Andrade ${ }^{(1)}$, Simone Pivaro Stadiniky ${ }^{(2)}$, \\ Eduardo Frota Carrera $^{(3)}$, Giany Gonze Tellini ${ }^{(4)}$
}

\section{RESUMO}

Objetivos: os objetivos deste estudo foram traduzir a versão original da avaliação funcional de ombro Modified-University of California at Los Angeles Shoulder Rating Scale da língua inglesa para a portuguesa e adaptar culturalmente à população brasileira. Métodos: a escala foi traduzida e adaptada culturalmente para a população brasileira de acordo com a metodologia internacionalmente aceita descrita por Guillemin et $a^{28}{ }^{28}$. A versão traduzida e revisada pelo comitê foi aplicada em dois grupos (indivíduos leigos com idade entre 45 e 75 anos e profissionais da saúde) para avaliação do nível de compreensão das alternativas. Encontrando $15 \%$ ou mais de alternativas "não-compreendidas", os termos foram substituídos por palavras equivalentes de modo que não fossem alterados conceito, estrutura e propriedade de base do instrumento, e reaplicadas até que valores menores que $15 \%$ fossem alcançados. Resultados: foram necessárias três aplicações. Cada grupo continha 20 indivíduos selecionados consecutivamente, totalizando 120 pessoas. Para obtenção da versão final, foram modificadas cinco alternativas do domínio dor e quatro alternativas do domínio função. Além dessas alterações, foram identificados problemas, por ambos os grupos, quanto à estrutura da escala, sugerindo a continuação do estudo de sua validade e possíveis modificações desta.

Palavras-Chave: ombro doloroso, tradução, adaptação cultural, Modified-UCLA.

\begin{abstract}
Objectives: the aims of this study were to translate into BrazilianPortuguese the Modified - University of California at Los Angeles Shoulder Rating Scale and to cross-culturally adapt it to the Brazilian environment. Methods: the scale was translated to Portuguese and back translated into English according to internationally recommended process described by Guillemin et al ${ }^{(28)}$. The translated and revised version was administered to two groups (patients between 45 and 75 years old and health professionals) to evaluate the comprehension level of the items. If $15 \%$ or more of health professionals or patients reported difficulty in understanding, that item was reformulated and reapplied. Results: we did three applications. Each group had 20 consecutive people, totalizing 120 people. To obtain the final Brazilian version, we modified five items in the pain parameter and four in the function parameter. Beyond these alterations, we identified problems about the structure suggesting that we need to continue the validity and possible modifications of this scale.
\end{abstract}

Keywords: shoulder painful, translation, cross-cultural adaptation, Modified-UCLA. 


\section{INTRODUÇÃO}

Os instrumentos para avaliação funcional do ombro são propostos como um método simplificado para avaliar e comparar o tratamento de várias alterações do ombro ${ }^{(1-16)}$.

Alguns instrumentos, inicialmente, propuseram avaliar a resposta de certos procedimentos em um grupo específico de pacientes. Outros foram criados para serem usados de forma genérica para diversas disfunções do ombro. Dessa forma, ainda não existe um instrumento de avaliação padronizado para ser utilizado em nosso meio ${ }^{(16)}$.

Os instrumentos freqüentemente utilizados na literatura internacional para avaliação do ombro são: o University of California at Los Angeles (UCLA) Shoulder Rating Scale, Modified University of California at Los Angeles (modified UCLA) Shoulder Rating Scale, Scoring System of ConstantMurley, American Shoulders and Elbow Surgeons (ASES), Shoulder Pain and Disability Index (SPADI), Simple Shoulder Test (SST). No Brasil, o Modified - UCLA tem sido o mais usado ${ }^{(17-25)}$, no entanto, este instrumento ainda não foi adaptado para o nosso contexto cultural.

A UCLA Shoulder Rating Scale foi originalmente descrita por Amstutz et a ${ }^{(26)}$, em 1981, para avaliar pacientes que se submetiam à artroplastia de ombro. É composta de três domínios: dor, função e força muscular e movimento que somam 30 pontos. Quanto maior a pontuação, melhor os resultados. Em 1986, foi modificada por Ellman et a ${ }^{(27)}$ e utilizada para avaliação pré e pós-operatória de lesões degenerativas do manguito rotador.

A UCLA-modificada (Anexo l) é composta pelos seguintes domínios: dor (10 pontos), função (10 pontos), amplitude da flexão anterior ativa ( 5 pontos $)$, teste de força manual para flexão anterior ( 5 pontos) e satisfação do paciente ( 5 pontos), somando 35 pontos no total. Os itens dor, função (em atividades de vida diária) e satisfação do paciente são avaliados por meio de questionamentos e os itens amplitude da flexão anterior ativa e teste de força manual para flexão anterior, por meio de exame físico rotineiro, o que torna a escala de fácil aplicação e extremamente viável para acompanhamento em clínicas, consultórios ou até mesmo ambiente hospitalar ${ }^{(27)}$.

A pontuação é classificada da seguinte forma: 34-35 pontos correspondem a resultados excelentes, 28-33 bons, 21-27 razoáveis e 0-20 ruins. Neste mesmo estudo, abdução e rotação externa também foram avaliadas, porém considerou-se apenas a flexão anterior com a justificativa de ser um movimento essencial para realização das atividades acima do nível do ombro e atrás da cabeça. Os pesquisadores também citaram a importância da avaliação da dor e força, visto que são fatores altamente incapacitantes,
ANEXo 1

MODIFIED - UNIVERSITY OF CALIFORNIA AT LOS ANGELES SHOULDER RATING $S_{C A L E}(28)$

PAIN

1) Present all of the time and unbearable; strong medication frequently

2) Present all of the time but bearable; strong medication occasionally

2

3) None or little at rest, present during light activities salicylates frequently

4) Present during heavy or particular activities only; salicylates occasionally

5) Occasional and slight 8

6) None 10

\section{FUNCTION}

\begin{tabular}{ll}
\hline 1) Unable to use limb & 1 \\
2) Only light activities possible & 2 \\
3) Able to do light housework or most activities of daily living & 4 \\
$\begin{array}{l}\text { 4) Most housework, shopping, and driving possible; able to do hair } \\
\text { and dress and undress, including fastening brassiere }\end{array}$ & 6 \\
$\begin{array}{l}\text { 5) Slight restriction only; able to work above } \\
\text { shoulder level }\end{array}$ & 8 \\
\hline 6) Normal activities & 10 \\
\hline
\end{tabular}

\section{ACTIVE FORWARD FLEXION}

\begin{tabular}{ll}
\hline 1) 150 degrees or more & 5 \\
2) 120 to 150 degrees & 4 \\
\hline 3) 90 to 120 degrees & 3 \\
4) 45 to 90 degrees & 2 \\
5) 30 to 45 degrees & 1 \\
6) Less than 30 degrees & 0 \\
\hline
\end{tabular}

STRENGTH OF FORWARD FLEXION (MANUAL MUSCLE-TESTING)

\begin{tabular}{ll}
\hline 1) Grade 5 (normal) & 5 \\
2) Grade 4 (good) & 4 \\
3) Grade 3 (fair) & 3 \\
4) Grade 2 (poor) & 2 \\
4) Grade 1 (muscle contraction) & 1 \\
6) Grade 0 (nothing) & 0 \\
\hline
\end{tabular}

SATISFACTION OF THE PATIENT

\begin{tabular}{ll}
\hline 1) Satisfied and better & 5 \\
2) Not satisfied and worse & 0 \\
\hline
\end{tabular}


ou seja, pacientes com dor e fraqueza importantes, neste estudo, tiveram maiores chances de evoluir com resultados razoáveis ou ruins, além da insatisfação pessoal ${ }^{(27)}$.

Sendo assim, a escala modificada apresentou duas alterações: a divisão do domínio força muscular e movimento em dois domínios de avaliação física, e a adição do domínio satisfação aumentando em cinco pontos o total da escala.

Considerando a freqüente citação da escala UCLA-modificada na literatura brasileira, e sendo esta um instrumento de origem estrangeira, fica explícita a necessidade de uma tradução confiável e adaptação cultural para o país ou região em que vem sendo aplicada.

Os objetivos do estudo foram: (1) traduzir a versão original da avaliação funcional de ombro modified-UCLA da língua inglesa para a portuguesa e (2) adaptar culturalmente à população brasileira.

\section{MATERIAL E MÉTODOS}

Para as etapas de tradução e adaptação cultural, seguiuse as diretrizes propostas por Guillemin et $a^{(28)}$.

Inicialmente, foram realizadas duas traduções da versão original americana para o português (tradução inicial) por dois professores brasileiros com proficiência em língua inglesa e previamente esclarecidos sobre o objetivo do estudo. As duas traduções foram comparadas e as divergências modificadas para um consenso quanto à tradução inicial.

Em seguida, a primeira versão traduzida foi encaminhada ao comitê formado por dois ortopedistas especialistas em ombro e dois fisioterapeutas especializados em ortopedia, vinculados ao Lar Escola São Francisco (LESF) e ao Hospital São Paulo (HSP) com conhecimento da língua inglesa e portuguesa, dos objetivos da pesquisa e da escala. Esse comitê avaliou a qualidade do material e sugeriu possíveis atualizações ou modificações dos termos.

A versão revisada em português foi convertida para o inglês ( back-translation) por dois professores de inglês nascidos nos Estados Unidos que não conheciam os objetivos nem a versão original. Essas duas novas versões foram comparadas com o instrumento original em inglês e as discrepâncias existentes documentadas e analisadas pelo mesmo comitê até obtenção de uma segunda versão em português.

Para a adaptação cultural, esta foi aplicada em dois grupos: (1) grupo formado por pessoas com idade entre $45 \mathrm{e}$ 75 anos, alfabetizadas ou não, sem conhecimento sobre o assunto e que não apresentavam qualquer queixa associada às lesões no ombro e, (2) grupo de profissionais da saúde que conheciam o assunto de estudo para verificar a compreensão dos mesmos em relação ao enunciado e às alternativas de resposta de cada questão. Ambos os grupos eram vinculados à Escola Paulista de Medicina da Universidade Federal de São Paulo (UNIFESP) e provenientes do LESF e do HSP.

Foram aplicadas apenas as alternativas dos domínios dor, função e satisfação do paciente da versão traduzida da UCLA-modificada, pois os outros domínios são itens de avaliação física (teste de força e goniometria). Para a padronização da aplicação da escala, foram elaboradas instruções para que o entrevistado respondesse unicamente o que the foi perguntado.

Apesar de as respostas serem mais fidedignas ao entregar a escala, optamos pela leitura das alternativas para evitar a exclusão de indivíduos que não sabiam ler e/ou com problemas visuais.

Após a aplicação, as alternativas eram classificadas em "compreendida" e "não-compreendida" pelos indivíduos. As alternativas que apresentaram índice de 15\% ou mais de "não-compreendida" foram reescritas e substituídas por alternativas equivalentes de modo que não fossem alterados conceito, estrutura e propriedade de base do instrumento.

Uma vez realizada as aplicações nos dois grupos, as questões que deveriam ser modificadas eram reunidas em uma única versão, levando em conta às considerações da população leiga e dos profissionais e o nível de escolaridade dos grupos onde prevaleceu o grupo com menor nível de instrução para que a última versão fosse compreendida por todos.

Sendo assim, uma vez modificadas as alternativas, o questionário foi reaplicado a um novo grupo de indivíduos leigos $(n=20)$, e um novo grupo de profissionais $(n=20)$ sendo necessárias três reaplicações em cada grupo $(n=120)$, até que nenhuma alternativa fosse classificada com $15 \%$ ou mais de "não-compreendida".

\section{RESULTADOS}

Em relação ao grupo 1, a média de idade foi de 55,6 anos $(\mathrm{DP}=8,98)$. Nas três aplicações, houve maior freqüência da faixa etária de 45 a 55 anos (55\%) e o nível de escolaridade predominante foi o $1^{\circ}$ grau incompleto $(53,33 \%)$.

No processo de tradução, durante as revisões das versões pelo comitê, obtivemos algumas mudanças como a dos títulos: "atividade de flexão para frente" e, "força de flexão para frente (teste manual do músculo)" para "flexão anterior ativa" e "força de flexão anterior (teste de força manual)", respectivamente. O teste de força muscular manual é caracterizado pela aplicação da resistência manual do examinador a fim de determinar a capacidade dos músculos ou grupos musculares de funcionar em movimento e sua habilidade para prover estabilidade e suporte. A graduação baseia-se 
na habilidade de manter a parte testada em uma posição contra a gravidade podendo ser classificado em: 0 (ausência de contração muscular), l (contração muscular mas, sem movimento), 2 (contração muscular com movimento eliminando a ação da gravidade), 3 (movimento contra a ação da gravidade), 4 (movimento contra uma pequena resistência) a 5 (movimento contra uma grande resistência) ${ }^{(29,30)}$.

Alguns termos como "esporadicamente" e "certas atividades" foram igualmente modificados por termos equivalentes como "ocasional" e "atividades específicas".

Também foram alterados termos em inglês durante a back-translation como constant por present all of the time.

Finalizado o processo de tradução, foi iniciado o processo de adaptação cultural, no qual as alternativas com $15 \%$ ou mais para "não-compreendidas" foram modificadas e adaptadas até que valores menores que $15 \%$ fossem alcançados.

Em relação ao domínio dor, conforme observamos nos Gráficos 1 e 2, na primeira aplicação, as alternativas que obtiveram índice de "não-compreensão" de $15 \%$ ou mais foram $1,2,3$, 4 e 5 .

Nas alternativas 1 e 2 , a palavra "forte", relacionada ao tipo de medicamento, foi discutida em relação aos parâmetros do autor de classificar em fraco e forte, já que o paciente não conseguiu distinguir esta classificação; o mesmo ocor-

GRÁFICO 1

Porcentagem de QUeSTÕes NÃO COMPREENDIDAS PELOS PROFISSIONAIS RELACIONADAS AO DOMÍNIO DOR

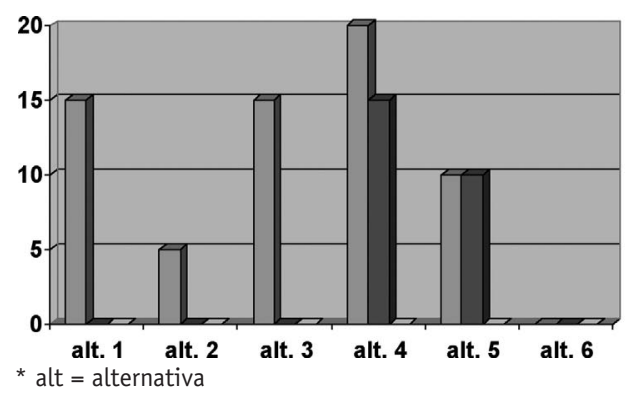

GRÁFICO 2

PorcentageM DE QUESTÕES NÃO COMPREENDIDAS PELA POPULAÇ̃̃o LEIGA RELACIONADAS AO DOMÍNIO DOR

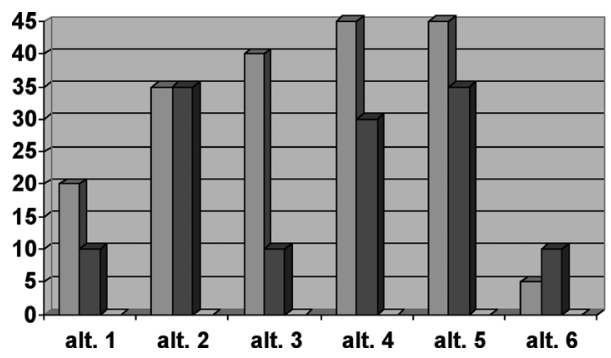

* alt = alternativa $\square$ aplicação 1

$\square$ aplicação 2

$\square$ aplicação 3

$\square$ aplicação 1

$\square$ aplicação 2

$\square$ aplicação 3 rendo com a palavra "antiinflamatórios não-hormonais", presente nas alternativas 3 e 4 . Como a questão do uso do medicamento vem dentro de um contexto que inclui vários parâmetros (freqüência e intensidade da dor, tipo e freqüência do uso de medicamento), achamos melhor substituí-las apenas pela palavra medicação, não alterando a resposta do indivíduo, em termos de pontuação da escala.

Nas alternativas 1, 2, 3 e 5, os termos "freqüentemente", "ocasionalmente" e "ocasional" foram substituídos por palavras equivalentes para que não alterassem o conceito, a estrutura e a propriedade de base do instrumento.

$\mathrm{Na}$ alternativa 3, o termo "repouso" foi entendido como "dormindo", sendo que esta palavra foi substituída por "quando o braço está parado".

Nas alternativas 3 e 4, o termo "atividades" foi entendido como atividade física (esporte) sendo trocada por "trabalho".

Em relação ao domínio função, apresentado nos Gráficos 3 e 4, na primeira aplicação, as alternativas que obtiveram índice de "não-compreensão" de $15 \%$ ou mais foram $1,3,4$ e 5 .

$\mathrm{Na}$ alternativa 1 , o termo "membro" foi considerado um termo técnico pelo grupo de leigos, sendo substituído por "braço".

GRÁFICO 3

Porcentagem de QUeSTÕes NÃO COMPREENDIDAS PELOS PROFISSIONAIS RELACIONADAS AO DOMÍNIO FUNÇÃO

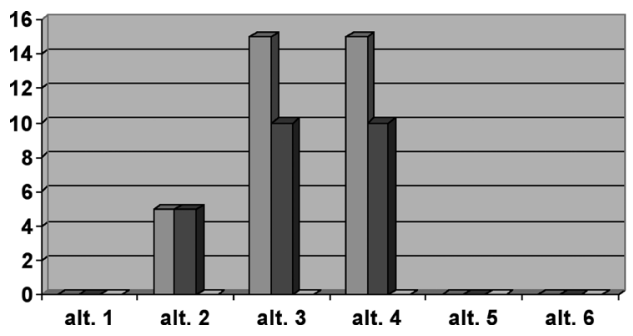

$\square$ aplicação 1

$\square$ aplicação 2

$\square$ aplicação 3

* alt $=$ alternativa

GrÁfico 4

Porcentagem de QUeSTÕes NÃO COMPREENDIDAS PELA POPULAÇÃO LEIGA RELACIONADAS AO DOMÍNIO FUNÇÃO

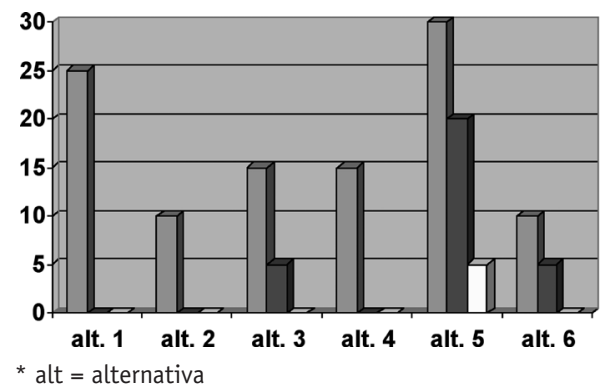

$\square$ aplicação 1

$\square$ aplicação 2

$\square$ aplicação 3 
$\mathrm{Na}$ alternativa 3, o termo "leves" foi acrescentado, pois na tradução e revisão da última versão em inglês para o português a palavra "leves" foi omitida. Além disso, a palavra "atividades" foi substituída por "trabalhos".

$\mathrm{Na}$ alternativa 4, a frase foi reescrita trocando a ordem das palavras, mas mantendo o conceito e a estrutura, de forma que houvesse compreensão desta.

$\mathrm{Na}$ alternativa 5, os termos "restrições" e "nível" não foram entendidos pela população leiga, sendo substituídos por "limitações" e "altura", respectivamente.

As alternativas do domínio satisfação tiveram $100 \%$ de compreensão tanto pelos leigos como pelos profissionais. Portanto, nenhum termo foi alterado.

$\mathrm{Na}$ segunda aplicação, apenas as alternativas modificadas desta versão foram reaplicadas. As alternativas modificadas 2 , 4 e 5 , do domínio dor, novamente obtiveram índice de "não-compreensão" nos termos "eventualmente" e "eventual", que foram substituídos por "de vez em quando", palavra sugerida tanto pelo grupo de pessoas leigas como de profissionais. Para o domínio função, apenas a alternativa 5 manteve índice insatisfatório. Sendo assim, somente as alternativas com necessidade de modificação foram reaplicadas.

Na terceira aplicação, obtivemos $100 \%$ de compreensão pelos profissionais em todas as alternativas. No entanto, para a alternativa 5 do domínio função houve $5 \%$ de não compreensão entre os indivíduos leigos. Esse valor não foi significativo para modificação, portanto, $o$ termo foi mantido.

Finalmente, foi obtida a versão traduzida e adaptada para a língua portuguesa da escala UCLA-modificada (Anexo 2).

\section{ANexo 2}

ESCALA TRADUZIDA E ADAPTADA À LINGUA PORTUGUESA Sistema de PONTUAČ̃̃ Da UCLA (Universidade da Califórnia - Los Angeles) DOR

\begin{tabular}{l}
$\begin{array}{l}\text { 1) Presente todo o tempo e insuportável; faz uso de medicação } \\
\text { regularmente }\end{array}$ \\
$\begin{array}{ll}\text { 2) Presente todo o tempo, mas suportável; faz uso de } \\
\text { medicação de vez em quando }\end{array}$ \\
$\begin{array}{ll}\text { 3) Nenhuma ou pouca dor quando o braço está parado, ocorre } \\
\text { durante trabalhos leves; faz uso de medicação regularmente }\end{array}$ \\
$\begin{array}{ll}\text { 4) Ocorre apenas durante trabalhos pesados ou durante trabalhos } \\
\text { específicos; faz uso de medicação de vez em quando }\end{array}$ \\
$\begin{array}{ll}\text { 5) Dor leve e ocorre de vez em quando } \\
\text { 6) Nenhuma }\end{array}$ \\
\hline
\end{tabular}

\section{FUNÇÃO}

\begin{tabular}{ll}
\hline 1) Incapaz de usar o braço & 1 \\
2) Capaz apenas de realizar atividades leves & 2 \\
$\begin{array}{l}\text { 3) Capaz de realizar trabalhos domésticos leves ou a maioria dos } \\
\text { trabalhos do dia-a-dia }\end{array}$ & 4 \\
$\begin{array}{l}\text { 4) Capaz de realizar a maioria dos trabalhos domésticos, } \\
\text { inclusive fazer compras, dirigir, pentear-se, vestir-se, despir-se }\end{array}$ & 6 \\
$\begin{array}{l}\text { e fechar o sutiã } \\
\text { 5) Apresenta pouca dificuldade, capaz de realizar movimentos }\end{array}$ & 8 \\
$\begin{array}{l}\text { acima da altura do ombro } \\
\text { 6) Atividades normais }\end{array}$ & 10 \\
\hline
\end{tabular}

\section{INSTRUÇÕES PARA GONIOMETRIA}

0 paciente deve se posicionar sentado com o membro ao lado do corpo e posição neutra. 0 examinador deve instrui-lo a elevar o braço 0 máximo possível sem compensações.

0 goniômetro será posicionado com braço proximal na linha média axilar do tórax e braço distal na linha médio lateral do úmero, sendo o eixo colocado próximo ao acrômio.

\section{FLEXÃO ANTERIOR ATIVA}

\begin{tabular}{ll}
\hline 1) 150 graus ou mais & 5 \\
2) 120 a 150 graus & 4 \\
\hline 3) 90 a 120 graus & 3 \\
4) 45 a 90 graus & 2 \\
5) 30 a 45 graus & 1 \\
6) Menos que 30 graus & 0 \\
\hline
\end{tabular}

\section{INSTRUÇÕES PARA O TESTE DE FORÇA MANUAL}

0 paciente deve se posicionar sentado com o membro ao lado do corpo e antebraço pronado, a seguir deve elevar o membro a 90 graus. 0 examinador devera instrui-lo a manter esta posição contra resistência que será aplicada na porção distal do úmero (acima do cotovelo).

\section{FORÇA DE FLEXÃO ANTERIOR ATIVA} (TESTE DE FORÇA MANUAL)

\begin{tabular}{ll}
\hline 1) Grau 5 (normal) & 5 \\
\hline 2) Grau 4 (boa) & 4 \\
3) Grau 3 (regular) & 3 \\
4) Grau 2 (fraco) & 2 \\
5) Grau 1 (contração muscular) & 1 \\
6) Grau 0 (ausência de contração) & 0 \\
\hline
\end{tabular}

SATISFAÇÃO DO PACIENTE

\begin{tabular}{ll}
\hline 1) Satisfeito e melhor & 5 \\
2) Insatisfeito e pior & 0 \\
\hline
\end{tabular}

\section{DISCUSSÃO}

As diferenças existentes entre as definições, crenças e comportamentos impõem que a utilização de um ins- 
trumento elaborado em outros contextos culturais seja precedida, além de uma tradução confiável, uma adaptação cultural para o país ou região em que vem sendo aplicado, de forma que mantenha os mesmos conceitos do original. Essa adaptação não se restringe apenas às situações que envolvem países e/ou idiomas distintos. Ajustes locais e regionais também requerem atenção. Em países com raízes culturais tão heterogêneas como o Brasil, a proposição de termos coloquiais típicos, facilmente aceitos e compreendidos em uma determinada região poderia não ser pertinente a outro.

Durante as três aplicações, foram apontadas sugestões pelas populações com o propósito de discutir possíveis dificuldades de aplicação do instrumento relacionadas à forma em que o mesmo foi elaborado. Notamos algumas dificuldades quanto à aceitação da população para o uso de determinados termos na construção das frases dos dois primeiros domínios. Termos, como a palavra "trabalhos", foram considerados inespecíficos por abrangerem uma variedade de situações diferentes, tornando-os passíveis de interpretação subjetiva.

No domínio dor, as primeiras alternativas são formadas por orações independentes. A primeira oração cita duração e intensidade da dor e a segunda cita como o uso de medicação é empregado. Se tais alternativas fossem desmembradas, haveria uma maior facilidade em associar o relato do paciente com as colocações do questionário.

Kirkley et $a^{(31)}$ afirmam que a presença de muitas variáveis numa mesma alternativa garante uma dificuldade de resposta adequada uma vez que o paciente pode ser impossibilitado de escolher uma alternativa apropriada entre as opções disponíveis.

Foi questionado qual o critério utilizado para diferenciar o termo "medicação forte" do termo "antiinflamatórios não-hormonais", considerando que o tipo de medicamento e a freqüência em que foi utilizado não são parâmetros adequados para classificar a intensidade da dor do paciente.

Quanto ao domínio satisfação do paciente, este seria aceitável somente durante a evolução do tratamento, sendo

\section{REFERÊNCIAS}

1. Fenlin JMJ, Chase JM, Rushton SA, Frieman BG: Tuberoplasty: creation of an acromiohumeral articulation-a treatment option for massive, irreparable rotator cuff tears. J Shoulder Elbow Surg 11: 136-42, 2002

2. Kim SH, Ha KI, Kim SH: Bankart repair in traumatic anterior shoulder instability: open versus arthroscopic technique. Arthroscopy 18: 755-63, 2002. dispensável em uma avaliação inicial. Além disso, há necessidade da criação de mais alternativas. Kirkley et $a^{l^{31)}}$ também relatam que o domínio de satisfação garante a utilização do instrumento apenas após uma intervenção, além de não mostrar alternativa para casos em que não há evolução.

Os parâmetros considerados para que tais avaliações físicas fossem inseridas na escala não foram questionados por nós. Porém, Kirkley et al ${ }^{(31)}$ citam que o instrumento compara dois itens subjetivos de avaliação de função (dor e função) com avaliação física. Estes podem não ser compatíveis para serem combinados na pontuação total. Ainda nesse estudo, declaram que há dúvida também sobre a atribuição dos pesos dos domínios, sendo que os critérios para a distribuição da pontuação não são evidenciados, concluindo que, quanto à formulação da avaliação proposta pela escala, esta não é necessariamente incorreta, apenas insustentável.

\section{CONCLUSÃO}

A escala UCLA-modificada é conhecida atualmente como um instrumento clínico e de pesquisa amplamente utilizado. Embora seja encontrada sua aplicação em publicações nacionais, não foi observado nenhum estudo quanto à sua tradução para a língua portuguesa e adaptação cultural brasileira.

A forma como a escala foi desenvolvida inicialmente, assim como sua modificação, não sustentam diretrizes para sua utilização. Mesmo com sua tradução adaptada para a cultura brasileira, fica explícita a necessidade da continuação do estudo de validade e análise da estrutura da escala de avaliação funcional do ombro UCLA-modificada, assim como uma possível modificação da estrutura proposta.

\section{AGRADECIMENTOS}

Ao Prof. Dr. Geraldo da Rocha Castelar Pinheiro pelo seu incentivo à pesquisa e publicação deste artigo.

Declaramos a inexistência de conflitos de interesse.
3. Castellanos G, Camilo E, Segura D, Jaime E, Lizcano OVH, Argote RA: Resultados del tratamiento del sindrome de pinzamiento subacronial sin lesión del manguito rotador: acromioplastia artroscópica versus acromioplastia abierta por técnica de "mini-incisión". Rev Colomb Ortop Traumatol 16: 25-35, 2002.

4. Arce G, Lacroze P, Butler S, Previgliano JP, Pereira E: Reconstruccion artroscopica de la inestabilidad anterior del 
hombro. Rev Asoc Argent Ortop Traumatol 67: 177-80, 2002.

5. Gartsman GM, Roddey TS, Hammerman SM: Arthroscopic treatment of multidirectional glenohumeral instability: 2 - to 5-year follow-up. Arthroscopy 17: 236-43, 2001.

6. Samani JE, Marston SB, Buss DD: Arthroscopic stabilization of type II SLAP lesions using an absorbable tack. Arthroscopy 17: 19-24, 2001.

7. Gartsman GM, Roddey TS, Hammerman SM: Arthroscopic treatment of bidirectional glenohumeral instability: two to five year follow-up. J Shoulder Elbow Surg 10: 28-36, 2001.

8. Montenegro S, Brañes M, Nuñez R: Pinzamiento anteromedial subcoracoideo. Rev Argent Artrosc 7: 20-4, 2000.

9. Romeo AA, Hang DW, Junior Bach BR, Shott S: Repair of full thickness rotator cuff tears. Gender, age, and other factors affecting outcome. Clin Orthop 367: 243-55, 1999.

10. Gartsman GM, Brinker MR, Khan M: Early effectiveness of arthroscopic repair for full-thickness tears of the rotator cuff: an outcome analysis. J Bone Joint Surg Am 80: 33-40, 1998.

11. Bosch U, Skutek M, Fremerey RW, Tscherne H: Outcome after primary and secondary hemiarthroplasty in elderly patients with fractures of the proximal humerus. J Shoulder Elbow Surg 7: 479-84, 1998.

12. Aparicio JL: Lesiones del manguito rotador del hombro: tratamiento: artroscopía + abordaje mínimo (mini open repair). Rev Asoc Argent Ortop Traumatol 63: 35-40, 1998.

13. Gartsman GM: Massive, irreparable tears of the rotator cuff. Results of operative debridement and subacromial decompression. J Bone Joint Surg Am 79: 715-21, 1997.

14. Carpignano R: Patología del espacio subacromial. Rev Asoc Argent Ortop Traumatol 61: 169-78, 1996.

15. Burns TP, Turba JE: Arthroscopic treatment of shoulder impingement in athletes. Am J Sports Med 20: 13-6, 1992.

16. Romeo AA, Bach Junior BR, O'Halloran KL: Scoring systems for shoulder conditions. Am J Sports Med 24: 472-6, 1996.

17. Checchia SL, Doneux P, Miyazaki NA, Pombo EH, Meirelles Júnior MS: Osteonecrose da cabeça do úmero: resultados das artroplastias. Rev Bras Ortop 36: 29-36, 2001.

18. Balsini N, Balsini NE: A cirurgia de descompressão subacromial do ombro por videoartroscopia: avaliação e resultados. Rev Bras Ortop 35: 127-30, 2000.

19. Godinho GG, Freitas JMA, Leite LMB, Pina ERM: Lesões SLAP no ombro. Rev Bras Ortop 33: 345-52, 1998.

20. Rodrigues SA, Lemos JA, Reis ML: Hemiartroplastia do ombro não cimentada com prótese global. Rev Bras Ortop 32: 703-6, 1997.

21. Volpon JB, Muniz AAS: Resultado do tratamento cirúrgico do pinçamento do manguito rotador do ombro pela descompressão subacromial. Rev Bras Ortop 32: 65-9, 1997.

22. Godinho GG, Souza JMG, Oliveira AC, Freitas JM: Artroscopia cirúrgica no tratamento da síndrome do impacto: nossa experiência em 100 casos cirúrgicos. Rev Bras Ortop 30: 540-6, 1995.

23. Brasil Filho R, Fillardi Filho CS, Menitti EL, Souza ASDE: Avaliação de pacientes operados devido à síndrome do pinçamento, com ou sem lesão do manguito rotador. Rev Bras Ortop 30: 649-54, 1995.

24. Checchia SL, Doneux SP, Martinez PE, Garcia SCM, Leal HP: Tratamento da capsulite adesiva do ombro pelo bloqueio do nervo supra-escapular, associado ao uso de corticóide. Rev Bras Ortop 29: 627-34, 1994.

25. Rodrigues SA, Fiuza LS, Louzada PHV: Transposiçäo do "latissimus dorsi" para tratamento das lesões maciças do manguito rotator. Rev Bras Ortop 29: 638-42, 1994.

26. Amstutz HC, Sew Hoy AL, Clarke IC: UCLA anatomic total shoulder arthroplasty. Clin Orthop 155: 7-20, 1981.

27. Ellman H, Hanker G, Bayer M: Repair of the rotator cuff. J Bone Joint Surg Am 68-A: 1136-44, 1986.

28. Guillemin F, Bombardier C, Beaton D: Cross-cultural adaptation of health-related quality of life measures: literature review and proposed guidelines. J Clin Epidemiol 46: 1417-32, 1993.

29. Kendall FP, McCreary EK, Provance PG: Músculos: provas e funções, $4^{\mathrm{a}}$. ed, São Paulo: Editora Manole, 1995.

30. Daniels L, Worthigham C: Muscle testing: techniques of manual examination, Philadelphia, WB Sauders, 1980.

31. Kirkley A, Griffin S, Dainty K: Scoring system for the functional assessment of the shoulder. Arthroscopy 19: 1109-20, 2003. 